\title{
Re-opening Exercise Science Laboratories and Testing During the Covid-19 Endemic Phase
}

Authors

Donald R. Dengel, Nicholas G. Evanoff

\section{Affiliation}

School of Kinesiology, University of Minnesota, Minneapolis

Key words

COVID-19, cardiopulmonary exercise testing, body

composition, decision making, strength testing

accepted 23.09.2020

published online 19.10 .2020

\section{Bibliography}

Int J Sports Med 2021; 42: 789-793

DOI 10.1055/a-1283-6256

ISSN $0172-4622$

(C) 2020. Thieme. All rights reserved.

Georg Thieme Verlag KG, Rüdigerstraße 14,

70469 Stuttgart, Germany

Correspondence

Dr. Donald R. Dengel

School of Kinesiology, University of Minnesota

111 Cooke Hall

55455 Minneapolis, MN

United States

Tel. : +16126269701, Fax : +16126258867

denge001@umn.edu

\begin{abstract}
The coronavirus disease 2019 (COVID-19) pandemic has forced primary/grade schools and university closings as well as forced the suspension of a number of medical and laboratory testing procedures. Exercise science laboratories whether in clinical, research or educational locations were also forced to pause testing procedures. As the COVID-19 pandemic begins to subside in some areas of the world, exercise science laboratories are contemplating how to create a safe environment to resume some laboratory testing activities. In this article, we present suggestions for how exercise science laboratories can open and create a safe environment for subjects, laboratory personnel and equipment upon reopening.
\end{abstract}

\section{Introduction}

A number of clinical, research and teaching exercise science laboratories involved in physiological and exercise testing as well as teaching were forced to close temporarily due to a highly infectious, severe acute respiratory syndrome coronavirus 2 (SARS-CoV-2) that is responsible for the coronavirus disease 2019 (COVID-19) pandemic. As COVID-19 pandemic begins to recess in certain areas of the world and testing services to the healthcare and sports communities are starting to resume activities, exercise science laboratories are attempting to develop protocols and procedures to create safe environments for testing a wide variety of the population. Before reopening exercise science laboratories, there are a number of things to consider.

It is important to remember that the COVID-19 virus is transmitted via respiratory droplet and contact route [1,2]. The simplest control measures to interrupt droplet and contact transmission is social distancing, or keeping at least 1.5-2 meters or more apart $[3,4]$ and implementing hand washing hygiene $[5,6]$. In addition, the spread of COVID-19 can be further reduced when face coverings are used along with these other preventive measures [7, 8]. Finally, the cleaning and disinfecting of frequently touched surfaces as well as equipment used in a laboratory will also greatly reduce the transmission of SARS-CoV-2 $[8,9]$.

Due to inherent social distancing limitations, no matter what type of testing is done in an exercise science laboratory; it will require the use of personal protective equipment (PPE). Therefore, the availability of PPE and clinical demand for PPEs will need to be factored into the consideration for resuming testing [10]. As PPE manufacturing increases to meet demand and the supply chain recovers from the current crisis, rational sourcing and use of PPEs can be implemented. If local healthcare services are lacking PPE or regional demand is high, it is recommended that procurement of PPE and, hence, opening exercise testing laboratories should be delayed to avoid competition with healthcare systems for PPEs [10].

Finally, the population being tested should also be considered. Older adults and people of any age who have serious underlying 
medical conditions (i. e. heart or lung disease, diabetes, etc.) seem to be at higher risk for developing more serious complications from COVID-19 illness [11,12]. Additionally, vulnerable researchers and technicians (e.g. age demographic, increased risk factors) should also be considered and those researchers should use caution when opting to participate in testing procedures that may increase their risk for COVID-19. Therefore, these general issues should be thoroughly examined and considered prior to the resumption of physiologic and exercise testing.

\section{General Testing Considerations}

When setting up a safe laboratory environment there are some general issues as well as some individual issues that are specific to certain physiologic and exercise tests that need consideration. Regardless of the type of testing, there are three areas of safety to consider: 1) Subject safety, 2) Test technician(s) safety, and 3) Equipment safety.

One general consideration to enhance both subject and test technician(s) safety is to pre-screen both prior to having them come into the laboratory for testing. Simple questions regarding whether individuals have experienced fever, chills, shortness of breath, cough, or loss of taste and smell within the past 7 days should be asked $[13,14]$. These questions should mirror local government guidelines since COVID-19 related symptoms may differ from region to region [15]. If answers to any of the questions are "yes" the subject should not be scheduled for testing and be referred to their medical provider for follow-up. In some cases such as in regions where COVID-19 is very prevalent, any signs of illness regardless of COVID-19 related symptoms might exclude the subject from visiting the laboratory.

Similarly, asking if, within the past 14 days, the individual has been exposed to someone with a known positive test for SARSCOV-2 or they themselves have tested positive for SARS-COV-2 should be implemented. Depending on the severity of COVID-19, an individual may be at higher risks for cardiovascular complications during exercise due to cardiac injury [16] or vascular damage [17]. These added risks should be taken into consideration and discussed with subjects prior to testing.

Checking an individual's body temperature before entering the laboratory may also be considered as an added safeguard. If the body temperature is equal to or less than $37.4^{\circ} \mathrm{C}$ they can enter the lab. If a person's temperature is equal to or greater than $37.4^{\circ} \mathrm{C}$ they should not enter the laboratory and local guidelines for seeking medical advice and possible testing for SARS-COV-2 should be followed $[2,18,19]$.

Laboratory mechanical ventilation flow rates and circulation should also be considered before opening an exercise science laboratory (especially if high ventilation exercise is occurring such as maximal aerobic capacity $\left(\mathrm{VO}_{2 \max }\right)$ assessments. The American Society of Heating, Refrigerating and Air-Conditioning Engineers (ASHRAE) states ventilation and filtration can reduce the concentration of SARS-COV-2 in the air [20]. Most healthcare settings have adequate system requirements to provide proper ventilation [21]. If ventilation rates of an exercise science laboratory are insufficient, other means such as opening windows/doors to allow natural ventilation, creating a negative pressure chamber, or implementing portable air disinfection devices may be beneficial [21, 22]. Since exercise science laboratory environments are diverse each laboratory should take ventilation into consideration when opening and planning testing capacity.

Another factor to consider is minimizing possible exposure and spread to SARS-COV-2 is to limit number of people. This can be accomplished by allowing only the minimum number of technicians needed for each testing procedures while ensuring emergency protocols will be easily implemented if needed. Many institutions limit space capacity at $50 \%$ of total occupancy and this number should be posted at the entrance of the lab per institutional or community regulations.

The last general consideration before opening an exercise science laboratory is how to deliver lifesaving procedures, if needed, due to cardiac complications during exercise. The general consensus outside of a healthcare setting is to follow general protocol for cardiopulmonary resuscitation: chest compressions and defibrillation without rescue breaths [23, 24]. Nevertheless, laboratory personnel should develop a plan in accordance to guidelines proposed by the laboratory's local health authorities.

\section{Subject Safety}

The main area to consider regarding the resumption of laboratory testing is the subject traffic flow into and out of your testing facility. Whenever possible, minimizing contact between subjects is warranted to maximize safety. This can be accomplished in a number of ways.

One is to lengthen the time between testing so that a subject will be gone from the laboratory before the next subject enters the laboratory for testing; another way to minimize subject contact is to provide separate entrance and exit areas. The second issue is to keep each subject's belongings together and isolated. This can be accomplished by providing each subject with a container such as a plastic bin that can be cleaned to place their personal items (i. e., coat, wallets, mobile phones, purses, backpacks, etc.). Following testing, subjects can take their items out of the bin and the bin can then be disinfected and readied for the next individual to use. The third issue is to have the individual being tested wear a facemask whenever possible. This will depend to some degree on the testing procedures involved. The rationale behind wearing a facemask to lower the transmission of the COVID-19 virus as well as other airborne pathogens is well documented in healthcare settings such as exercise science laboratories $[9,25,26]$. The final issue regarding subject safety issues is prior to having a subject enter the laboratory have them sanitize their hands. To accomplish this a hand sanitizing station should be placed at the entrance to the laboratory and signage asking subjects to use it before entering the laboratory should be visible

\section{Test Technician Safety}

The safety of the person(s) administering the test also needs to be considered. Like the subject being tested, the technician(s) administering the test should wear a facemask. For healthcare providers medical or surgical masks are recommended [27]. These facemasks are made to guarantee one-way protection for healthcare workers, in order to capture their respiratory droplets. For additional protection, a face shield should be used in conjunction with the facemask (discussed below) [28]. 
Given that the technician(s) of the laboratory test might have contact with the subject, the technician(s) of the laboratory test should wear exam gloves since they are going to need to touch the subject and equipment. Depending on the nature and volume of the tests, consider having the technician(s) of the test wear a disposable gown (discussed below).

\section{Equipment Safety}

The third area of creating a safe testing environment is to consider the equipment being used during laboratory testing. Obviously, the laboratory equipment will need to be cleaned and disinfected between subjects. Prior to the current COVID-19 pandemic this was considered good clinical practices and is even more important in the current situation. Whenever cleaning and disinfecting laboratory equipment you should refer to the manufacturer's official technical bulletin or User Guide. However, it is possible that any official documents regarding cleaning and disinfection of equipment may have been written prior to COVID-19 pandemic and may lack consideration of the issues surrounding COVID-19. Therefore, there are some general things to consider regarding equipment. The first thing to consider is that whatever is used to clean and disinfect the equipment that the agent you use needs to kill the COVID-19 virus.

There are a number of disinfectants (i. e., sprays, wipes, etc.) that can be used to eliminate the COVID-19 virus. The United States Environmental Protection Agency (EPA) provides an updated comprehensive list of sprays and wipes that kill the COVID-19 virus [29]. Besides the brand; manufacturer; and active ingredients, the list provides surface type effectiveness. In addition, the list also provides the contact time for each product, which is important to determine the time needed between subjects [29]. The choice of whether to use a wipe or a spray may depend somewhat on the surface being cleaned or disinfected. Care should be given when using any type of cleaner or disinfectant to make sure that it does not stain or discolor equipment. For instance, disinfectants that contain iodine may stain plastic, which is often used as outer housing on laboratory equipment. It is recommended that before using a disinfectant a small amount be placed on an inconspicuous area on the equipment housing. Wait a day and check to make sure that no stain or discoloration has occurred.

Some equipment in exercise science laboratories may need additional considerations to ensure they are operating as safely as possible. Below we list some other things to consider when testing during various sports, clinical and exercise testing.

\section{Cardiopulmonary Exercise Testing}

By far the most common test in exercise science laboratories is cardiopulmonary exercise testing. Cardiopulmonary exercise testing is often used to evaluate cardiorespiratory fitness; it is also frequently used in the assessment of pulmonary, cardiac and physical impairments since it accurately uncovers systemic abnormalities in a safe and reproducible manner [30,31]. Given that cardiopulmonary exercise testing involves an increase in pulmonary inspiration and expiration there is concern that cardiopulmonary exercise testing is an aerosol-generating procedure [32]. Therefore, if cardiopulmonary exercise testing is to be conducted some extra safeguards may need to be considered.

\section{Subject Safety}

Obviously, cleansing and sterilization of equipment is standard infection control practice, but it is now more important than ever in cardiopulmonary exercise testing. Whenever possible single-use masks, sensors, turbines, and gas lines should be used. However, when this is not possible, manufacturer approved disinfectants should be used on equipment that will be reused. It is recommended that exercise science testing facilities be left unused for a minimum of 20 min to allow airborne droplets to settle on surfaces before sterilizing and disinfecting equipment and surfaces [33]. Additionally, if possible, filters should be used during cardiopulmonary testing.

Breathing filters with $~ 99.9 \%$ efficiency against viruses are available. In addition, these breathing filters have been shown not to impede flow rates that achieved during cardiopulmonary exercise testing in the average population [33]. Most manufacturers of cardiopulmonary testing equipment have recommended filters. The location of breathing filters depends on the type of metabolic cart used and is discussed in detail in the section below.

\section{Test Technician Safety}

Due to the possible aerosol-generating issues surrounding cardiopulmonary exercise testing, we recommend a N95/FFP2 face mask for those individuals administering the cardiopulmonary exercise test. In addition, face shields and disposable gowns are also recommended for the individual administering the cardiopulmonary exercise test [34]. The breathing filters discussed above will help protect the technician from exposure to the concentrated flow that occurs with most commercial metabolic carts. However, maximizing distance from the exercising subject as well as possibly adding a barrier between the subject and technician should be considered in certain laboratory settings.

\section{Equipment Safety}

Breathing filters not only offer protection to the subject and technician, but also offer a degree of protection to the cardiopulmonary testing equipment. Cardiopulmonary metabolic carts that utilize a mixing chamber typically place a filter proximal to the mixing chamber just distal to the water collecting apparatus. This will protect the mixing chamber from contamination. Adding another breathing filter to the intake portion of the two way non-rebreathing valve can protect the participant from droplet inhalation. For inline pneumotach cardiopulmonary metabolic carts a breathing filter may be placed directly distal to the mouthpiece, which can filter the inspired air as well as the expired air. Prior to using a breathing filter it is best to consult the cardiopulmonary testing equipment manufacturer's manual when selecting a filter as well as determining the best placement of the breathing filter.

\section{Body Composition Testing}

Outside of cardiopulmonary exercise testing, examination of body composition is the second most common form of testing done in most exercise science laboratories. Body composition can involve very simple equipment such as tape measures and calipers to more complex equipment such as dual X-ray absorptiometry (DXA). Given the wide variety of equipment used some extra safeguards 
may need to be considered during testing for body composition depending on the equipment used.

\section{Subject Safety}

One may wonder about having a subject wear a mask during body composition testing especially if using a DXA. There is no issue concerning having a mask on the face (cloth or paper) altering the DXA scan results. Anyone who has undergone a DXA scan realizes that the subject will touch the DXA scanner and the scanner table to help positioning themselves. So having subjects wash their hands prior to getting on the scanner can help lower the possibility of the individual contaminating the DXA scanner.

Determining body composition using hydrostatic weighing systems which involves the use of a water tank should be used with extra precautions such as proper water treatments and caution with forced expiratory practices in enclosed spaces. If hydrostatic weighing systems are to be used, local guidelines should be followed similar to public pools $[35,36]$. Similarly, air displacement plethysmography body composition systems should also be used with caution. Since subjects are asked to breathe in an enclosed space and masks may interfere with accuracy, so filtering of inspired and expired air should be considered. In addition, the measuring pod needs to be thoroughly cleaned and disinfected between subjects.

Body composition measurements done using skinfold calipers or circumference take relies on direct close contact with subjects. Care should be used with these types of testing due to the duration and proximity of collecting accurate measurements Therefore, the above safeguards (gloves, face mask, etc.) should be used.

\section{Test Technician Safety}

Given that the test Technician of most body composition will be in close contact with the subject to some degree, it is recommended that they wear both gloves, face mask. A face shield should be considered as well.

\section{Equipment Safety}

All of the equipment used in body composition testing including weight scale, calipers, stadiometer, tape measures, etc. need to be clean and disinfected between subjects.

\section{Anaerobic/Strength Testing}

Anaerobic testing such as Wingate or strength testing often involve maximal effort and the possibility of maximal forced expirations. Therefore, anaerobic/strength testing offers different challenges to creating a safe testing environment.

\section{Subject Safety}

Unlike cardiopulmonary exercise testing where subjects breathe through a mask or mouthpiece, subjects involved in anaerobic and strength testing do not. Therefore, it is recommended that the subject wear a face covering during testing to help lower respiratory droplet formation.

\section{Test Technician Safety}

Similar to the precautions used for the technician(s) of cardiopulmonary exercise testing, administrators of anaerobic and strength testing should wear the appropriate PPEs (i. e., N95/FFP2 face mask, gown, shield, etc.). In addition, creating a minimum distance of 1.5 to 2 meters between the subject and the technician(s) of the test is recommended, whenever possible. Strength testing (i. e. 1 repetition maximal tests) should be done using a machine so the administrator can avoid close contact during "spotting" that occurs with free weights.

\section{Equipment Safety}

All of the equipment used in anaerobic/strength testing including machine, weights, weight bar, etc. need to be clean and disinfected between subjects

\section{Conclusion}

We hope this article provides some insights into creating a safe testing and laboratory environment for both the subject as well as the tester. Given all of these changes, exercise physiologists will need to make adjustments in the time normally scheduled for testing and be diligent in hygienic practices to ensure a safe and accurate testing environment. In conclusion, the above suggestions are good clinical practices and even with the hopeful end of the COVID19 virus crisis, these practices should be followed in the future to ensure a safe exercise science laboratory.

\section{Conflict of Interest}

The Authors of this manuscript (D.D and N.E.) have no conflict of interest and this manuscript was built based on the ethical standards of the International Journal of Sports Medicine [37].

\section{References}

[1] Chavez S, Long B, Koyfman A et al. Coronavirus Disease (COVID-19): A primer for emergency physicians. Am J Emerg Med 2020; Online ahead of print, Im Internet: http://dx.doi.org/10.1016/j.ajem.2020.03.036

[2] World Health Organization. Modes of transmission of virus causing COVID-19: implications for IPC precaution recommendations. World Health Organization; 2020 Im Internet: https://www.who.int/ news-room/commentaries/detail/modes-of-transmission-of-viruscausing-covid-19-implications-for-ipc-precaution-recommendations

[3] Mittal R, Ni R, Seo J-H. The flow physics of COVID-19. J Fluid Mech. 2020; 894: Im Internet: https://www.cambridge.org/core/journals/ journal-of-fluid-mechanics/article/flow-physics-of-covid19/476E32549 012B3620D2452F30F2567F1

[4] Singh N, Kaur M. On the airborne aspect of COVID-19 coronovirus. arXiv [physics.pop-ph]. 2020; Im Internet: http://arxiv.org/ abs/2004.10082

[5] Basseal JM, Westerway SC, McAuley T. COVID-19: Infection prevention and control guidance for all ultrasound practitioners. Australas ] Ultrasound Med 2020; 23: 90-95

[6] Rundle CW, Presley CL, Militello M et al. Hand Hygiene During COVID-19: Recommendations from the American Contact Dermatitis Society. J Am Acad Dermatol. 2020; Online ahead of print, Im Internet: http://dx.doi.org/10.1016/j.jaad.2020.07.057

[7] Chu DK, Akl EA, Duda S et al. Physical distancing, face masks, and eye protection to prevent person-to-person transmission of SARS-CoV-2 and COVID-19: a systematic review and meta-analysis. Lancet 2020; 395: 1973-1987 
[8] Fathizadeh H, Maroufi P, Momen-Heravi M et al. Protection and disinfection policies against SARS-CoV-2 (COVID-19). Infez Med 2020; 28: 185-191

[9] National Center for Immunization and Respiratory Diseases (NCIRD). About Masks. Centers for Disease Control and Prevention 2020; Im Internet: https://www.cdc.gov/coronavirus/2019-ncov/prevent-getting-sick/about-face-coverings.html

[10] Livingston E, Desai A, Berkwits M. Sourcing Personal Protective Equipment During the COVID-19 Pandemic. JAMA 2020; Im Internet: http://dx.doi.org/10.1001/jama.2020.5317

[11] Zheng Z, Peng F, Xu B et al. Risk factors of critical \& mortal COVID-19 cases: A systematic literature review and meta-analysis. J Infect 2020; 81: e16-e25

[12] Division of Population Health, National Center for Chronic Disease Prevention and Health Promotion. COVID-19 Guidance for Older Adults. Centers for Disease Control and Prevention. 2020; Im Internet: https://www.cdc.gov/aging/covid19-guidance.html

[13] de Oliveira Neto L, de Oliveira Tavares VD, Schuch FB et al. Coronavirus pandemic (SARS-COV-2): Pre-exercise screening questionnaire (PESQ) for telepresential exercise. Front Public Health 2020; 8: 146

[14] Fu L, Wang B, Yuan T et al. Clinical characteristics of coronavirus disease 2019 (COVID-19) in China: A systematic review and metaanalysis. J Infect 2020; 80: 656-665

[15] Rothan HA, Byrareddy SN. The epidemiology and pathogenesis of coronavirus disease (COVID-19) outbreak. J Autoimmun 2020; 109: 102433

[16] Mitrani RD, Dabas N, Goldberger J]. COVID-19 cardiac injury: Implications for long-term surveillance and outcomes in survivors. Heart Rhythm. 2020; Online ahead of print, Im Internet: http://dx.doi. org/10.1016/j.hrthm.2020.06.026

[17] Marone EM, Rinaldi LF. Upsurge of deep venous thrombosis in patients affected by COVID-19: Preliminary data and possible explanations. J Vasc Surg Venous Lymphat Disord 2020; 8: 694-695

[18] Occupational Safety and Health Administration. Guidance on Preparing Workplaces for COVID-19 US Department of Labor. 2020; Im Internet: https://www.dol.gov/newsroom/releases/osha/ osha20200309

[19] WHO. Transmission of SARS-CoV-2: Implications for Infection Prevention Precautions. World Health Organization; 2020; Im Internet: https://www.who.int/emergencies/diseases/novel-coronavirus-2019/technical-guidance-publications

[20] ASHRAE Board of Directors. ASHRAE Position Document on Infectious Aerosols 2020; Im Internet: https://www.ashrae.org/file\%20library/ about/position\%20documents/pd_infectiousaerosols_2020.pdf

[21] Morawska L, Tang JW, Bahnfleth W et al. How can airborne transmission of COVID-19 indoors be minimised? Environ Int 2020; 142: 105832

[22] Cubillos ], Querney ], Rankin A et al. A multipurpose portable negative air flow isolation chamber for aerosol-generating procedures during the COVID-19 pandemic. Br J Anaesth 2020; 125: e179-e181

[23] Edelson DP, Sasson C, Chan PS et al. Interim guidance for basic and advanced life support in adults, children, and neonates with suspected or confirmed COVID-19: From the emergency cardiovascular care committee and get with the guidelines ${ }^{\circledR}$-Resuscitation adult and pediatric task forces of the American Heart Association in Collaboration with the American Academy of Pediatrics, American Association for Respiratory Care, American College of Emergency Physicians, The Society of Critical Care Anesthesiologists, and American Society of Anesthesiologists: Supporting Organizations: American Association of Critical Care Nurses and National EMS Physicians. Circulation 2020; 141: e933-e943; Im Internet: https://www.ahajournals.org/doi/ abs/10.1161/CIRCULATIONAHA.120.047463
[24] Nolan JP, Monsieurs KG, Bossaert L et al. European Resuscitation Council COVID-19 guidelines executive summary. Resuscitation 2020; 153: 45-55

[25] Maclntyre CR, Chughtai AA. Facemasks for the prevention of infection in healthcare and community settings. BMJ 2015; 350: h694

[26] Maclntyre CR, Chughtai AA. A rapid systematic review of the efficacy of face masks and respirators against coronaviruses and other respiratory transmissible viruses for the community, healthcare workers and sick patients. Int J Nurs Stud 2020; 108: 103629

[27] Ferioli M, Cisternino C, Leo V et al. Protecting healthcare workers from SARS-CoV-2 infection: practical indications. Eur Respir Rev 2020; 29: 200068. Im Internet: http://dx.doi.org/10.1183/16000617.0068-2020

[28] Lindsley WG, Noti JD, Blachere FM et al. Efficacy of face shields against cough aerosol droplets from a cough simulator. J Occup Environ Hyg 2014; 11: 509-518

[29] United States Environmental Protection Agency. List N: Disinfectants for Use Against SARS-CoV-2 (COVID-19). United States Environmental Protection Agency. 2020; Im Internet: https://www.epa.gov/ pesticide-registration/list-n-disinfectants-use-against-sars-cov-2-covid-19

[30] Guazzi M, Bandera F, Ozemek C et al. Cardiopulmonary exercise testing: What is its value? J Am Coll Cardiol 2017; 70: 1618-1636

[31] Christle JW, Arena R. Cardiopulmonary exercise testing and prescription of exercise. In: Pressler A, Niebauer J, Eds. Textbook of Sports and Exercise Cardiology. Cham: Springer International Publishing; 2020: 897-912

[32] Association for Respiratory Technology and Physiology COVID19 Group. COVID19 Infection Control Issues For Lung Function. ARTP. 2020; Im Internet: https://www.artp.org.uk/News/covid19-infectioncontrol-issues-for-lung-function

[33] Faghy MA, Sylvester KP, Cooper BG et al. Cardiopulmonary exercise testing in the COVID-19 endemic phase. Br J Anaesth 2020; 125 : 447-449. Im Internet: http://dx.doi.org/10.1016/j.bja.2020.06.006

[34] Matt O'Brien KM. NEW UPDATES: Restarting Your Lab: a pathway to resuming diagnostic testing (recording). 2020; Im Internet: https:// mgcdiagnostics.com/events/webinars

[35] World Health Organization. Water, Sanitation and Health Team. Guidelines for safe recreational water environments. Volume. 2: Swimming pools and similar environments. 2006; Im Internet: https:// apps.who.int/iris/handle/10665/43336

[36] National Center for Immunization and Respiratory Diseases (NCIRD), Division of Viral Diseases. Considerations for Public Pools, Hot Tubs, and Water Playgrounds During COVID-19 Centers for Disease Control and Prevention. 2020; Im Internet: https://www.cdc.gov/ coronavirus/2019-ncov/community/parks-rec/aquatic-venues.html

[37] Harriss D], MacSween A, Atkinson G. Ethical standards in sport and exercise science research: 2020 update. Int J Sports Med 2019; 40: 813-817 